Check for updates

Cite this: RSC Adv., 2017, 7, 34086

\title{
Progress in polydimethylsiloxane-modified waterborne polyurethanes
}

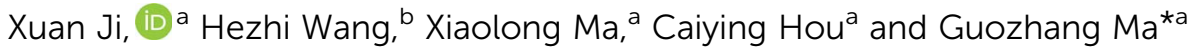

Preparing PDMS-modified WPU has attracted the attention of many researchers for two decades and this modification strategy has been proved to be an effective and feasible way to improve some key properties of WPU. In this review, both physical and chemical modification are introduced in depth. For chemical modification, the types of PDMS used, the copolymerization methods, the sub-classification of PDMS-modified WPU, and the synthesis processes are categorized and reviewed respectively; the effects of PDMS on colloidal, film-forming, surface, mechanical, and thermal properties as well as the effects of PDMS on microphase separation and hydrogen bonding behaviors of WPU are discussed in detail, with focus on the introduction to and understanding of the "structure-morphology-property" relationship of these hybrid materials. Besides, a current strategy to combine the PDMS modification technique with other modification methods to obtain novel WPU materials with superior properties is introduced. Finally, the challenges and future perspectives of this field are discussed.

Received 22nd May 2017 Accepted 14th June 2017

DOI: $10.1039 / \mathrm{c} 7 \mathrm{ra05738e}$

rsc.li/rsc-advances substrates (e.g., textile, metal, plastic, and wood). ${ }^{17,18}$ However, some properties of WPU still need to be further improved to meet various demands, such as water and solvent resistance, thermal stability, and mechanical strength. In this context, in order to overcome some drawbacks of WPU, numerous technologies have been developed over the past decade, such as copolymerizing or grafting of other polymers, ${ }^{19-21}$ rendering external or internal crosslinkings, ${ }^{22,23}$ simple blending or forming interpenetrating networks (IPNs), ${ }^{24}$ and modifying with nanoparticles. ${ }^{25-27}$

On the other hand, polydimethylsiloxane (PDMS) has many applications due to its unique properties, which arise mainly from its natural structure composed of inorganic Si-O bond and organic graft methyl group. These interesting properties include low surface energy, biocompatibility, high thermal stability, excellent water and oxidation resistance, chemical inactivity, good electric insulation, low glass transition temperature $\left(T_{\mathrm{g}}\right)$, great molecular flexibility, and so on. ${ }^{28-33}$ Preparing PDMS-modified WPU so as to combine the advantages of PDMS with those of WPU has attracted the attention of many researchers for a long time, and moreover, this modification strategy has been proved to be an effective and feasible way to improve some key properties of WPU. Considering the fast and ongoing development in this area, a timely and specific review is much needed. Accordingly, this review is provided to categorize and summarize the recent advances in PDMS-modified WPU, with focus on the introducing and understanding the "structure-morphology-property" relationship of these hybrid materials. The challenges and future perspective of the PDMS-modified WPU industry are discussed as well. 


\section{Physical modification}

Physical modification, including simply blending different polymers as well as forming interpenetrating networks (IPNs), is a common method to modify a polymer material by forming polymer mixtures that are linked together through secondary forces other than covalent bonds.

Over the past few decades, physical modification of bulk or solvent-based PUs by PDMS has been studied in detail and a brief review is provided here so as to give a better understanding about the physical modification of WPU by PDMS. Due to the incompatibility between PU and PDMS, simply blending PU with PDMS is totally unsatisfactory and distinct phase separation is observed. Formation of IPNs is an effective method in overcoming the difficulty in mixing chemically incompatible polymers-IPNs are a class of blend where a polymeric network is synthesized in the presence of another previously or simultaneously established network. In this context, Ebdon et al. reported the synthesis and characterization of polyether urethane-PDMS IPNs. ${ }^{34}$ Vlad et al. also reported the synthesis and characterization of IPNs obtained by combination of castor oil-based PU with PDMS.$^{35}$ According to their studies, because of the large difference in solubility parameters between the PU network $\left[19.4 \times 10^{3}\left(\mathrm{~J} \mathrm{~m}^{-3}\right)^{1 / 2}\right]$ and PDMS network $\left.\left[14.7 \times 10^{3}\left(\mathrm{~J} \mathrm{~m}^{-3}\right)^{1 / 2}\right]\right]^{34-36}$ these IPNs were highly incompatible and exhibited high degree of phase separation. In order to improve the miscibility of the abovementioned PU-PDMS systems, several attempts were made, including (a) using poly(phenylmethylsiloxane) (PPMS) instead of PDMS, which is reasonable because PPMS has a more similar solubility parameters $\left[18.6 \times 10^{3}\left(\mathrm{~J} \mathrm{~m}^{-3}\right)^{1 / 2}\right]$ to PU network, ${ }^{37}(\mathrm{~b})$ adding compatibilizers, such as PDMS- $g$-PU, ${ }^{35}$ and (c) replacing either the pure PDMS or pure PU with a PU-co-PDMS network, expecting both networks having chemically identical segments might enhance the degree of mixing. ${ }^{38}$ Though a better mixing between PU and PDMS was demonstrated by these methods, however, these systems still showed gross phase separation and the expected excellent properties of the resulting materials which would combine the advantages of PU and PDMS were not reached. Physical modification of aqueous PU emulsions by PDMS was also attempted. In 1971, Klempner et al. combined the aqueous emulsions of both PU and PDMS with their crosslinking agents and stabilizers, forming a partial topologically IPNs. ${ }^{39}$ It should be noted here that, the aqueous hydrophobic PU emulsions used in the study were obtained by emulsification of PUs in water under strong shear forces in the presence of suitable external emulsifiers, which was a common practice prior to the invention of the superior ionomer-based WPU emulsions. The homogeneous mixtures were then cast into films and cured. The mechanical properties and solvent resistance of the resulting films, instead of showing any improvements, were much worse than those of the arithmetic means of each component, showing a limited degree of interpenetration and high incompatibility between PU and PDMS.

Compared with the above-discussed solvent-based and hydrophobic PUs, WPU has stronger polarity due to the incorporation of ionic moieties or of hydrophilic soft segments; thus, the polarity difference between WPU and PDMS is larger than that between PU and PDMS, which means the incompatibility problem for physical modification of WPU by PDMS would be even worse. For this reason, simply blending PDMS or modified PDMS with WPU has only been studied by few researchers. Yen et al. used PDMS as the only soft segment to synthesize a PDMS-based WPU (PWPU) and then blended it in different amounts with various types of WPU; a series of interdependent studies was conducted to investigate the effect of the PWPU on the solution properties, surface structure, and membrane properties of the blended WPU. ${ }^{40-43}$ According to their research, by blending a small amount of PWPU into WPU, the hydrophobic PDMS would migrate to the surface of the formed membranes, and therefore, the contact angle of water to the WPU membrane was increased greatly and immediately. ${ }^{42}$ The X-ray analysis shown that blending of PWPU to WPU would change the crystallizing properties of the WPU; hence, for some certain kinds of WPU with a certain amount of PWPU blended in, the thermal and physical properties of the blended films were significantly enhanced. ${ }^{41}$ Besides, Yen et al. also compared the surface structure and thermal properties of the formed membranes prepared by blending PWPU with WPU and by mixed soft-segment copolymerization; ${ }^{43}$ it was found that PDMS migrated to the surface much more easily in the blending method than in the copolymer method, and for the blending method, glass-transition and melting temperature increased rapidly when a small amount of PWPU was added into the WPU. The preliminary application of these blends (with small changes in composition) on the treatment of nylon fabrics was studied; ${ }^{40}$ the results shown that, after the treatment, the application properties of nylon fabrics were improved in comparison with those of the untreated fabric as well as the neat WPU treated fabrics.

\section{Chemical modification}

To date, the majority of researches, with the aim to increase the compatibility from the molecular level, have focused on chemical modification by introducing PDMS as soft segments into WPU structure. Generally, reactive PDMS are used in combination with other polyols (such as polyether or polyester), both serving as the soft segments of the PDMS-modified WPU (PDMS-WPU). Nonetheless, aqueous anionic WPU dispersions employing PDMS as the only soft segments were also prepared from hydroxylalkyl- or hydroxylether-terminated PDMS, aliphatic isophorone diisocyanate (IPDI), and dimethylolpropionic acid (DMPA).$^{44}$ The preliminary results shown that the coating properties of the air-dried, transparent films, highlighted in good adhesion to steel and excellent low temperature flexibility, were similar to those of the typical WPUs, whereas some mechanical properties such as elongation at break still needed much improvement.

\subsection{Types of reactive PDMS for the modification of WPU}

Currently, there are mainly four types of reactive PDMS used in the chemical preparation of WPU, i.e., PDMS with hydroxyl 
function, aminoalkyl function, hydroxyalkyl function, and hydroxypolyether function. These reactive PDMS, often with difunctionality, are used for the synthesis of block PDMS-WPU copolymers. In consideration for the requirements of the crosslinking density, cost, and application properties of the final product, the molecular weight of these PDMS generally ranges from 1000 to 4000 (with 2000 in particular). Table 1 gives the simplified structure of these reactive PDMS and the corresponding references about their applications in the modification of WPU.

Among these types of PDMS, hydroxyl-terminated PDMS is the cheapest and has been employed in many research studies; however, due to the easy hydrolysis of the $\mathrm{Si}-\mathrm{O}-\mathrm{C}$ bond that is formed between $\mathrm{Si}-\mathrm{OH}$ and NCO group, the hydrolytic stability of these PDMS-WPU copolymers in water is poor and this could be a major cause limiting their applications in industrial scale. ${ }^{45}$ Aminoalkyl-terminated PDMS has been rarely used in preparing the PDMS-WPU copolymer for the reason of (a) the high reactivity of $\mathrm{NH}_{2}$ group towards NCO group which may lead to explosive polymerization as well as gelation afterwards, and (b) the two reactive hydrogen in $\mathrm{NH}_{2}$ group which may react with NCO group to form cross-linked structure that would be difficult to disperse in water. Difunctional hydroxyalkyl-terminated PDMS and hydroxypolyether-terminated PDMS, having advantages in both forming $\mathrm{Si}-\mathrm{C}-\mathrm{O}$ bond which is more stable in water and having moderate reactivity over NCO group, are the commonly-used and promising types of PDMS in preparing PDMS-WPU copolymers. Besides, it should be noted here that the hydroxypolyether-terminated PDMS, in comparison with other types of PDMS, has better compatibility with WPU and their formed WPU-PDMS films are transparent, thereby making it suitable for the coating and other applications where high transparency is required. ${ }^{\mathbf{4 6 , 4 7}}$

\subsection{Block and graft copolymerization}

The above-mentioned reactive PDMS are generally used to prepare block PDMS-WPU in which the PDMS moieties are blocked along the polymer backbone; on the other hand, some

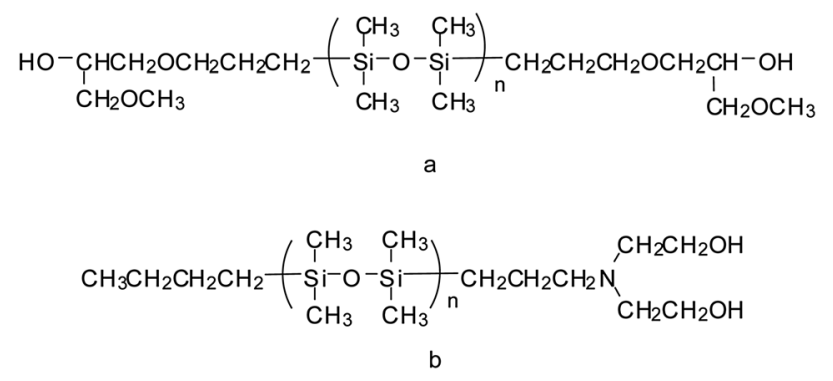

Fig. 1 Chemical structure of PDMS used to prepare block (a) and graft (b) PDMS-modified WPU. ${ }^{48}$

researchers have synthesized new reactive PDMS with a diol end group and then used it to prepare graft PDMS-WPU in which the PDMS moieties are grafted from the polymer backbone as side chains. For instance, Yu and Wang synthesized block and graft PDMS-WPU copolymer dispersions which were modified with $\alpha, \omega$-bis(3-(1-methoxy-2-hydroxypropoxy)propyl)-PDMS and $\alpha-N, N$-dihydroxyethylaminopropyl- $\omega$-butyl-PDMS, respectively (Fig. 1). ${ }^{48}$ The experimental results showed that PDMS could improve the water resistance property effectively regardless of the way of introducing the PDMS segment. Furthermore, the water absorption of the graft PDMS-WPU film $(17.3 \%)$ was lower than that of block PDMS-WPU film (40.2\%) with the same percent of PDMS $(5 \%, \mathrm{w} / \mathrm{w})$, and this is probably because that for graft copolymer films, the PDMS moieties were easier to migrate to the copolymer surface than those blocked to the copolymer backbone and therefore more hydrophobic PDMS chains migrated on the copolymer surface than blocked ones with the same percent of PDMS. This explanation was in agreement with the increasing degrees of microphase separation for WPU, block PDMS-WPU (BWPU), and graft PDMS-WPU (GWPU), as observed by their scanning electron microscope (SEM) images (Fig. 2). ${ }^{49}$ From these SEM images it was shown that the cross section of neat WPU was smooth, which indicates good compatibility among each component; the cross section of BWPU was rather coarse, which indicates that certain phase separation exists; for GWPU, a clearly PDMS enrichment region

Table 1 The structure and references for different type of reactive PDMS

\begin{tabular}{|c|c|c|}
\hline Type of reactive PDMS & Structure & Reference \\
\hline Hydroxyl-terminated & 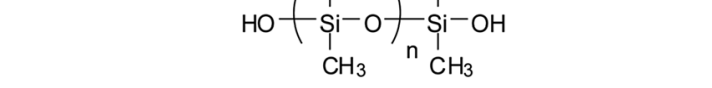 & $50-55$ \\
\hline Aminoalkyl-terminated & $\mathrm{H}_{2} \mathrm{~N}-\mathrm{R}-\underset{\mathrm{CH}_{3}}{\mathrm{Si}}+\underset{\mathrm{n}}{\mathrm{C}}{ }_{\mathrm{CH}_{3}}^{\mathrm{Si}}-\mathrm{R}-\mathrm{NH}_{2}$ where: $\mathrm{R}=$ =alkyl group & 56 \\
\hline Hydroxyalkyl-terminated & $\left.\mathrm{HO}-\mathrm{R}-\underset{\mathrm{CH}_{3}}{\mathrm{Si}}-\mathrm{O}\right)_{\mathrm{n}}{ }_{\mathrm{CH}_{3}}^{\mathrm{Si}}-\mathrm{R}-\mathrm{OH}$ where: $\mathrm{R}=$ alkyl group & $44,45,57$ and 58 \\
\hline Hydroxypolyether-terminated & 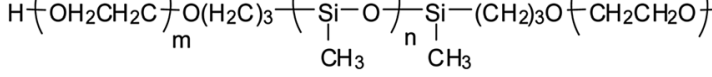 & $40-43,46$ and 47 \\
\hline
\end{tabular}



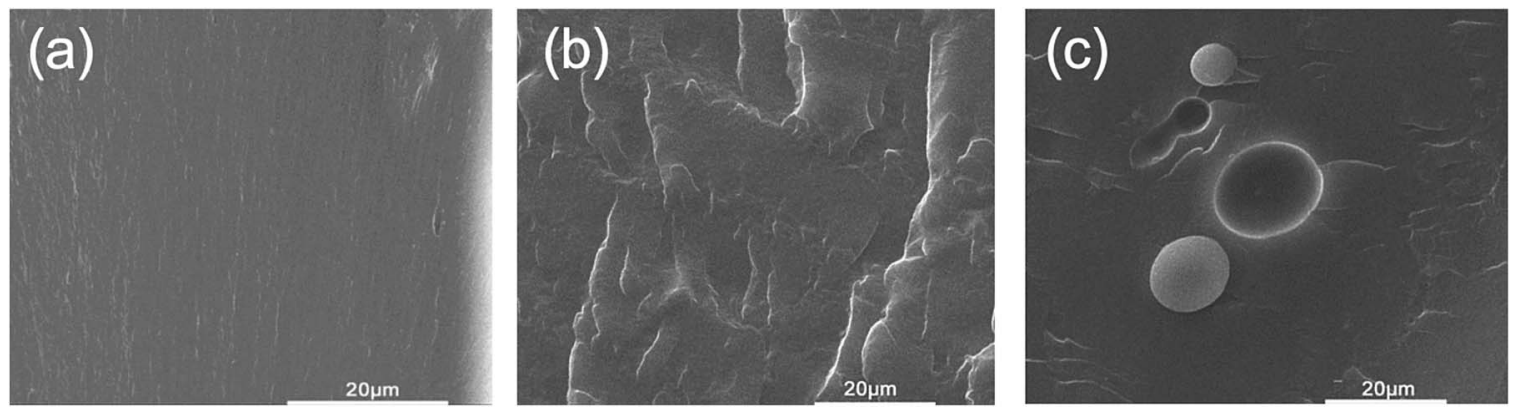

Fig. 2 SEM images for WPU films (a), BWPU film (b), and GWPU film (c). ${ }^{49}$

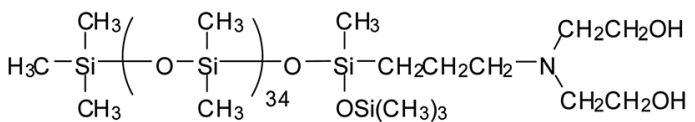

Fig. 3 Chemical structure of PDMS with a diol end group.

was observed (white balls), which indicates a high degree of microphase separation. Chen et al. reported a similar work to synthesize block and graft PDMS-WPU dispersions and then investigated their properties. In their study, when used in nylon fabric coating/dipping, the PDMS-WPU dispersions could increase the water repellency performance, indicating their potential applications in textile finishing. ${ }^{.9}$ Naghash et al. also reported the synthesis and characterization of a monotelechelic PDMS with a diol end group (Fig. 3) and then used it to modify WPU by graft copolymerization. ${ }^{60}$ The prepared graft PDMSWPU dispersion was applied as a potential marine coating and its antifouling property was investigated by immersion test under a marine environment. After 90 days, the fouled area was 98\% and 6\% for WPU and for PDMS-WPU (PDMS $4.48 \mathrm{wt} \%$ ), respectively. The reason for the good foul-release performance of PDMS-WPU was ascribed to the smooth coating surface and low surface energy (caused by PDMS), and low modulus.

With the same concept of polymer molecular design to make PDMS-grafted WPU, Li and Yue used aminoethylaminopropyl PDMS (AEAPPDMS) to synthesize a series of graft PDMS-WPU emulsions. ${ }^{61}$ In addition to the improvement in water resistance, the mechanical strength of the films was also increased with certain content of AEAPPDMS because of the cross-linking effect of the amino group. In order to improve the compatibility between AEAPPDMS and WPU, Wu et al. used a polyether-modified aminosilicone oil to replace AEAPPDMS and then synthesized graft PDMS-WPU emulsions. ${ }^{62}$ The mechanical, thermal, and icemelting stability of the emulsions prepared from the polyethermodified PDMS was higher than that of the emulsions prepared from AEAPPDMS; as for their formed films, the tensile strength and elongation at break were only fallen to a small extent as compared with non-modified WPU, all of which implied the compatibility between PDMS and WPU was indeed improved.

\subsection{Classification of PDMS-modified WPU}

Though a unified and acknowledged classification of PDMSWPU has not been established so far, the PDMS-WPU-like
WPU-can be classified according to a variety of different criteria, such as polymer structure, main raw materials used, synthesis methods, and the method or the field of its applications. ${ }^{16,18,63}$ In this review, depending upon the type of hydrophilic segments present in the polymer chain, the PDMS-WPU is classified as four types: anionic, cationic, zwitterionic, and nonionic.

Anionic PDMS-WPU is the most often studied materials in research articles; dimethylolpropionic acid (DMPA), which is a cheap and commercialized reagent, has been frequently used as an ionic diol and chain extender. ${ }^{17}$ The advantage of DMPA lies in the steric hinderance of the $\mathrm{COOH}$ group which prevents it from reacting with the NCO groups-this side reaction is not desirable because it could consume the potential ionic groups and cause high viscosities due to branching of the WPU. ${ }^{15}$

In contrast to the intensive studies about anionic PDMSWPU, there are few reported systematic studies on cationic PDMS-WPU. In this context, Wang et al. prepared a series of cationic PDMS-WPU dispersions and systematically studied the effects of PDMS content on the micromorphology, rheological behavior, and particle size of the dispersions, as well as the thermal properties, phase behavior, and surface structure of the PDMS-WPU films. ${ }^{53-55}$ According to their study, the particles had the morphology of a solid sphere, with particle size varying from $17.1 \mathrm{~nm}$ to $114.4 \mathrm{~nm}$ as the PDMS concentration increased from 0 to $25 \mathrm{wt} \%$. X-ray photoelectronic spectroscopy (XPS) spectra indicated the surface migration of Si element in the process of filming forming, and the film surface was mainly composed of soft segments. Differential scanning calorimeter (DSC) analysis, together with thermogravimetric analysis (TGA), differential thermogravimetry (DTG), and differential thermal analysis (DTA) results demonstrated the PDMS soft segment merged with the transition region of PU matrix, forming part of PU backbone, whereas an increased microphase separation was observed when PDMS concentration was greater than $15 \%$. It was also found that the incorporation of flexible PDMS prevented the degradation of WPU backbone, resulting in the increase of thermal stability in ultimate copolymer.

As far as we know, zwitterionic and nonionic PDMS-WPU have not been reported; future works are thus expected in view of the potential interesting properties these materials may possess. 


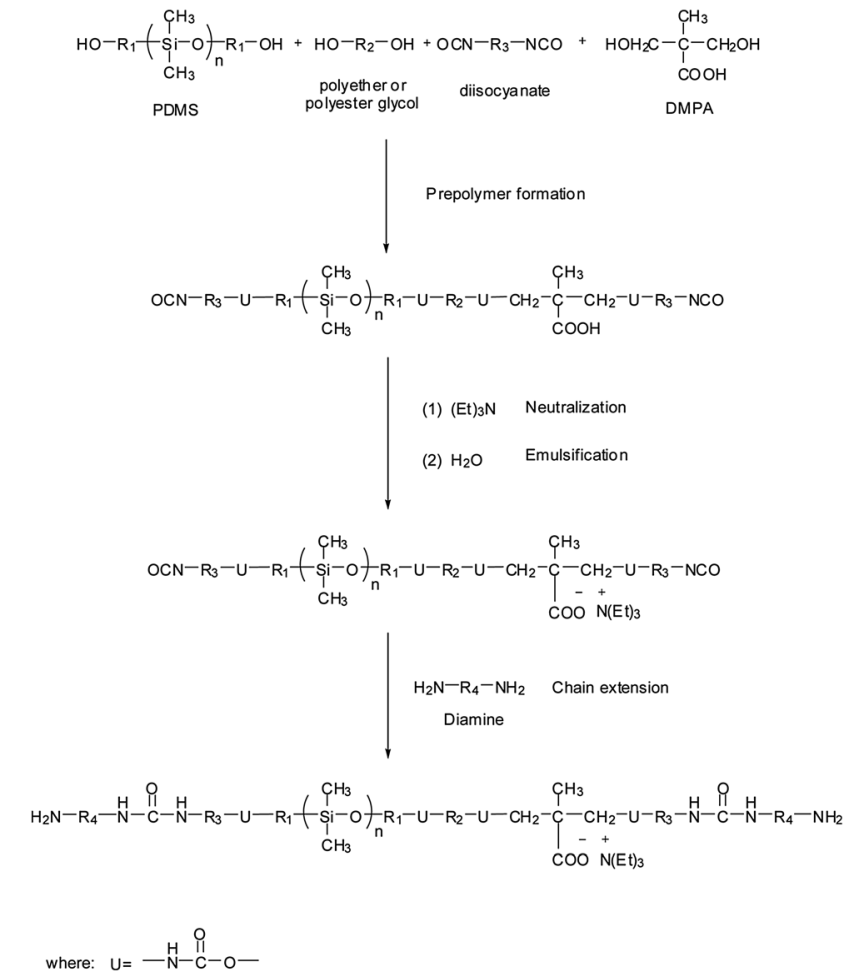

Fig. 4 Synthesis route of PDMS-modified WPU.

\subsection{Preparation process}

Over the past decades, various processes have been developed for the preparation of WPU dispersions. According to the differences in dispersing of prepolymer in water and the following chain extension step, these processes are categorized as four major types: prepolymer mixing process, acetone process, hot melt process, and ketimine-ketazine process. ${ }^{15,16,64,65}$ As for PDMS-WPU, the prepolymer mixing process has been the most widely adopted preparation method in industry and research. There are three basic steps in this process: (1) formation of isocyanate-terminated prepolymer by reacting long-chain polyols, reactive PDMS, diisocyanate, and hydrophilic agent; (2) neutralization of ionic groups by counter ions and emulsification of the NCO-terminated prepolymer for particle formation; (3) chain extension in the aqueous dispersion by reacting diamines with prepolymer for higher molecular weight and urea-linkage formation. ${ }^{51}$ A typical synthesis route is illustrated in Fig. 4.

\section{Effects of PDMS on properties of WPU}

\subsection{Effects of PDMS on colloidal properties of WPU}

The incorporation of PDMS has a major impact on various colloidal or solution properties of WPU dispersions, each of which would be discussed as follows.

The particle size of aqueous WPU dispersions, which can be varied from 0.01 to $5 \mu \mathrm{m}$, is important for its application and stability. ${ }^{15}$ For example, relative large particles are preferred in many surface coatings to facilitate rapid drying but are generally unstable with respect to sedimentation, while, relative small particles are more storage stable and are desirable when deep penetration of the dispersion into substrate is an essential step. The particle size is controlled by many factors, such as the structure and composition of WPU polymer, $\mathrm{pH}$ value, and emulsification conditions (temperature and $\mathrm{rpm}$ ).$^{17}$ In different researches, the average particle size of WPU dispersions would either increase or decrease with increasing PDMS content. ${ }^{\mathbf{5 6 , 6 0 , 6 6}}$ These contradictory results could be explained by different determining factors in play. For example, in the research of Fei et al., the particle size decreased with increasing PDMS content because the WPU chain length becomes short and tends to curl up; ${ }^{55}$ whereas in the research of Wang et al., when the WPU chain length remains the same but the PDMS content was increased by replacing other soft segments, the average particle size increased because the interfacial tension was increased with the addition of PDMS and this would lead to the increase of particle size according to emulsion polymerization theory and emulsion-forming equation. ${ }^{53,67}$

In general, the PDMS-WPU dispersions have relatively low viscosity with solid content $20-40 \%$. The viscosity of dispersion is affected by factors such as the interactions between particles, water swellability, and the particle size and its distribution.$^{67}$ Like the above-mentioned particle size, in different researches the viscosity also shows different trends with increasing PDMS content. For example, Yu and Wang reported that, with increasing PDMS content, the viscosity of the block PDMS-WPU dispersions was decreased but that of the graft ones was increased. ${ }^{48}$ The reason for this opposite change in viscosity is that for block PDMS-WPU the hydrophobic and flexible PDMS would reduce the interactions between segments but for graft PDMS-WPU the sterically hindered effect became dominant and led to the increase of particle interactions and thus viscosity.

Though the application conditions of WPU coatings are mainly determined by their rheological properties, the effect of PDMS on WPU's rheological properties was seldom studied. ${ }^{68}$ According to the research of Fei et al., pure WPU dispersions exhibit a Newtonian behaviour but PDMS-WPU dispersions are endowed with pseudoplasticity and thixotropy. ${ }^{55}$

In terms of other practical colloidal properties, it was found that the incorporation of PDMS would improve the levelling property of $\mathrm{WPU}^{57}$ but have a negative impact on properties associated with storage stability, such as freeze-thaw stability, centrifugal dispersion stability, and high temperature resistance. ${ }^{61,62}$ The poor storage performance arises from the poor compatibility between PDMS and WPU, and this problem could be partly solved by using limited amount of PDMS or employing polyether-modified PDMS to enhance the compatibility. ${ }^{46}$

\subsection{Effects of PDMS on film-forming and surface properties of WPU}

Generally, WPU dispersions exhibit excellent film-forming properties even at low temperature. ${ }^{15}$ The film-forming process for a typical WPU dispersion can mainly be divided into three stages: ${ }^{67}(1)$ the first stage is that with the evaporation of water, the 
emulsion particles are getting closer to each other to achieve a near-packed state. (2) The second stage is the deformation of emulsion particles. As the water further evaporates, the gap among particles becomes more and more narrow till they begin to contact, at the same time, the protective layer is damaged and the particles are gradually changed from spherical to hexahedron until the interface between them finally disappears. (3) The third stage is the diffusion process. The coil-shaped WPU polymers in emulsion particles are diffused and fused to each other and eventually forms a homogeneous film.

It is well known that the low surface energy of a component provides a thermodynamic driving force for migration to the polymer-air interface. When WPU is modified with PDMS by either chemical or physical method, hydrophobic PDMS with low surface energy and having different water solubility from WPU chain, during the film-forming process, will migrate and enrich at the interface of the emulsion and air, while some hydrophilic segments containing ionic or urethane groups will migrate and enrich at the interface of film and substrate. A possible film forming process of the PDMS-modified WPU is illustrated in Fig. 5. ${ }^{50}$ This surface enrichment of PDMS was confirmed by different researchers with various analysis techniques, such as XPS and attenuated total reflection Fourier transform infrared spectroscopy (ATR-FTIR). ${ }^{\mathbf{4 2 , 4 3 , 4 7 , 5 3 , 6 9}}$

The surface property of WPU films-which is a critical property in its many applications-would be dramatically improved due to the introduction of PDMS and its enrichment on the outmost surface of WPU-PDMS films. For instance, in the research of Li et al., as the content of PDMS increased from 0 to $25 \mathrm{wt} \%$, the surface free energy of the films decreased from 0.3446 to $0.2317 \mathrm{~J} \mathrm{~m}^{-2}$ and water absorption from $11.2 \%$ to $0.14 \%$, whereas the water contact angle increased from 75 to $96.5^{\circ}$, indicating the PDMS-WPU membrane surfaces have excellent water and chemical medium repellency. ${ }^{54}$ This improvement should be attributed to not only the hydrophobicity of PDMS enriched on the surface but also the enhanced surface roughness. ${ }^{50}$

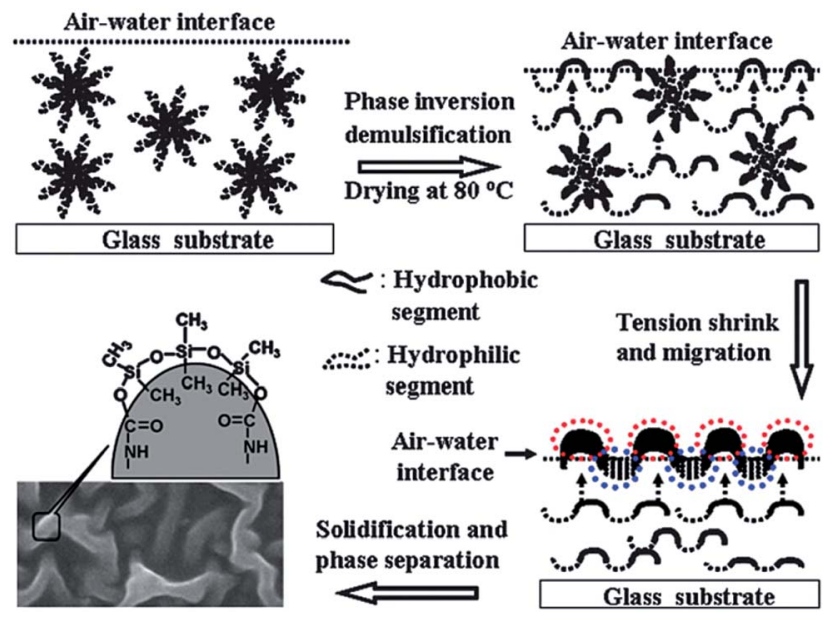

Fig. 5 A possible film forming process of the PDMS-modified WPU (reproduced from ref. 50 with permission from the Royal Society of Chemistry).

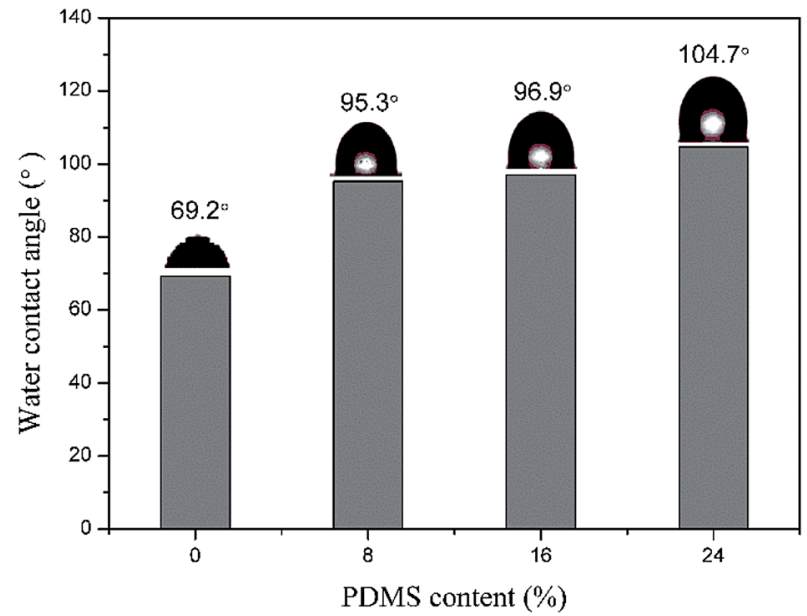

Fig. 6 Water contact angle of PDMS-modified WPU films (reproduced from ref. 66 with permission from the Royal Society of Chemistry).

Water absorption and water contact angle of WPU films, as two effective methods to measure the water resistance property, always decreases and increases with the increase of PDMS content (Fig. 6), respectively, implying the water resistance of WPU films is always improved with the increase of PDMS content. ${ }^{45,51,52,55,70,71}$ It is necessary to point out that water resistance of WPU films is always poor and inferior to the solvent-borne polyurethanes, which relates to the very nature of WPU (ionic groups in WPU ionomers are hydrophilic). ${ }^{\mathbf{1 5}}$ However, this problem has been effectively reduced or even resolved by the introduction of PDMS when compared with many other efforts, which thus becomes one main reason of employing PDMS to modify WPU.

\subsection{Effects of PDMS on microphase separation and hydrogen bonding of WPU}

WPU is segmented polymer which is composed of soft segments (stemming from polyols) and hard segments (stemming from diisocyanates and chain extenders) arranged alternatively. ${ }^{72}$ Generally, the hard segments can interact with each other through hydrogen bonds or other intermolecular forces and tend to agglomerate to form a compact rigid phase with high $T_{\mathrm{g}}$, whereas the soft segments would form the continuous soft matrix with a low $T_{\mathrm{g}}$ (Fig. 7). ${ }^{17,65}$ This microphase-separated morphology, as the most important feature of PU materials, makes a great contribution to its excellent performance: at service temperature, the soft phase is in a rubbery state endowing PU materials with elasticity; the hard phase, usually containing some crystallinity with sizes ranging from one to tens of nanometer, functions both as thermal reversible crosslink and as reinforcing filler to endow PU materials with mechanical strength, solvent resistance, and heat resistance. ${ }^{73}$ It is generally accepted that the hydrogen bonding between urethane units is the primary driving force for the hard domain aggregation and thus plays an important role in determining the microphase-separated morphology and its mechanical 


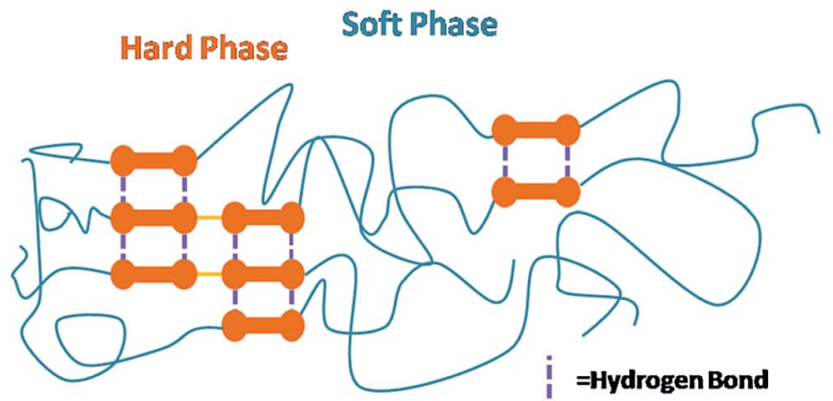

Fig. 7 Illustration of microphase separation in WPU (reproduced from ref. 72 with permission from Springer).

properties. ${ }^{15,16,63}$ FT-IR has been well established as an sensitive tool to study the hydrogen bonding both within hard segments and between soft/hard segments, thereby providing information about the degree of microphase separation. Other commonly-employed techniques in investigating microphase separation behaviour includes DSC, dynamic mechanical analysis (DMA), small angle X-ray scattering (SAXS), and wide angle X-ray scattering (WAXS). ${ }^{65,74}$

As the introduction of PDMS into WPU largely affects its microphase-separated morphology which determines to a large extent the ultimate properties of WPU, the study of the effects of PDMS on the degree of microphase separation lies in a central place in interpreting the structure-morphologyproperty relationship of WPU which is crucial to understand in the development and application of these materials. In theory, the phase separation, with the introduction of PDMS, should be enhanced obviously by the incompatibility of non-polar PDMS with low surface energy and high-polar hard segments, and as the content of PDMS increases the degree of phase separation should be further enlarged. This theoretical trend has been proved by many research results as the $T_{\mathrm{g}}$ values of soft segments (measured by DMA or DSC) shifted to lower temperatures where the $T_{\mathrm{g}}$ range of PDMS is -110 to $-129{ }^{\circ} \mathrm{C}$, while, the degree of hydrogen bonding (measured by calculating the degree of the urethane carbonyl groups participating in hydrogen bonding in FT-IR spectrum) increased as PDMS content in soft segments increased (Table 2 and Fig. 8). ${ }^{50,51,53,55,57,60,69}$ It should be noted that though the molecular weight and the content of PDMS have a big impact on the degree of phase separation, they had to be above a certain degree then the $T_{\mathrm{g}}$ values of soft segments can shift to lower temperature or a new $T_{\mathrm{g}}$ value which comes from PDMS could be observed. On the other hand, because the morphology of WPU is complicated and is controlled by numerous factors including the composition and the chemical structure of WPU chains, molecular weights and their distribution, linear or crosslinked structure, methods employed to apply the final product and process parameters adopted for that method and so on, ${ }^{\mathbf{1 8}}$ the effects of PDMS on the enhancement of microphase separation is not always guaranteed since the introduction of PDMS would probably change other impact factors. ${ }^{\mathbf{4 1 , 4 6 , 5 2 , 5 5}}$
Table 2 Experimental results of DSC for the PDMS-modified WPU films

\begin{tabular}{llll}
\hline Designation & PDMS concentration $^{a}(\%)$ & $T_{\mathrm{g} 1}\left({ }^{\circ} \mathrm{C}\right)$ & $\Delta C_{\mathrm{p}}\left(\mathrm{J}\left(\mathrm{g}^{-1} \mathrm{~K}^{-1}\right)\right)$ \\
\hline PU & 0 & - & - \\
SiPU-5 & 5 & - & - \\
SiPU-15 & 15 & -84.1 & 0.157 \\
SiPU-20 & 20 & -94.4 & 0.237 \\
SiPU-25 & 25 & -106.5 & 1.261
\end{tabular}

${ }^{a}$ The mass concentration of PDMS in PDMS-modified WPU (based on the total solid mass).

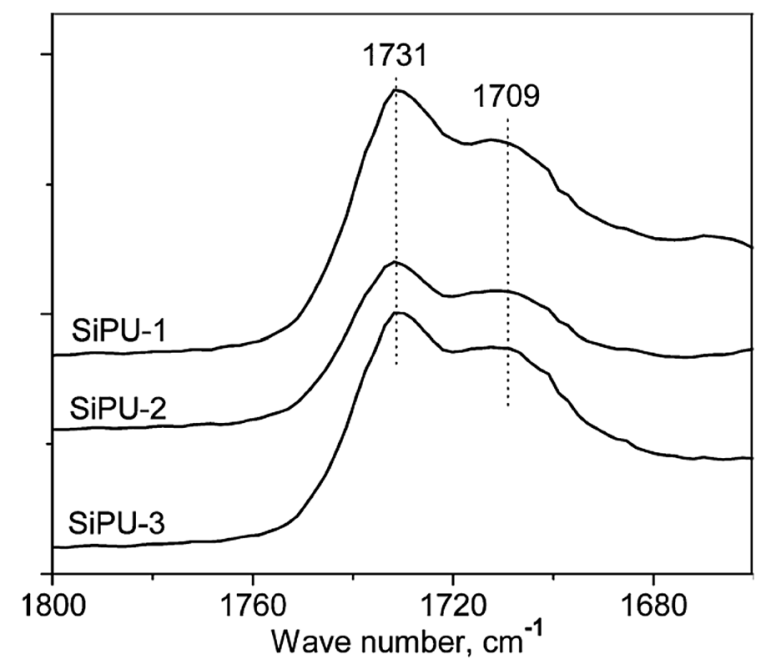

Fig. 8 FT-IR spectra of urethane $\mathrm{C}=\mathrm{O}$ stretching region of PDMSmodified WPU (reproduced from ref. 50 with permission from the Royal Society of Chemistry). The PDMS content in soft segments for SiPU-1, SiPU-2, and SiPU-3 is $50 \%, 60 \%$, and $67 \%$, respectively.

\subsection{Effects of PDMS on mechanical and thermal properties of WPU}

As stated above, the foundation of the versatility of PU materials is their microphase-separated morphology, and their mechanical properties-which range from flexible film to rigid elastomer-could be widely tuned by this feature as well as other synthetic parameters such as the molar ratio of hard/soft segments. ${ }^{18}$ The incorporation of PDMS often has a negative effect on the mechanical properties of WPU films because (a) the flexible and soft PDMS polymer with low molecular weight demonstrates poor mechanical properties and (b) the intermolecular forces between hard and soft segments (such as hydrogen bonding) is reduced due to the higher phase separation caused by PDMS. ${ }^{45,48,52,54,61,67}$ However, in some researches, the mechanical properties of PDMS-WPU films (like tensile strength and Young's modulus) improved slightly with the inclusion of little amount of PDMS but dropped down rapidly when more PDMS was introduced. 66,69,74 $^{\text {This change was }}$ explained that for mechanical properties only appropriate microphase separation is beneficial but excessive one is disadvantageous. ${ }^{51}$ Therefore, it is important to use optimum PDMS 


$$
\begin{aligned}
& \text { Potential post-crosslinking site }\left(\mathrm{HO}_{-}\right.
\end{aligned}
$$

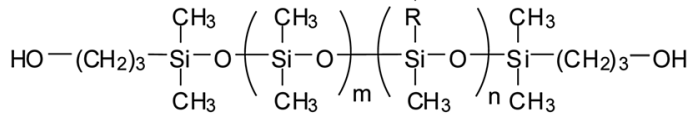

$$
\begin{aligned}
& \text { where: } \mathrm{R}=-\left(\mathrm{CH}_{2}\right)_{3}-\mathrm{O}-\stackrel{\mathrm{O}}{\mathrm{O}}-\mathrm{CH}_{2}-
\end{aligned}
$$

Fig. 9 Chemical structure of reactive PDMS bearing carbonyl groups.

content to obtain the proper mechanical properties. Besides, in order to further improve the mechanical properties of PDMSWPU films, crosslinking has been rendered into the polymer structure through either post-added crosslinking agent or preparing the internally crosslinked prepolymer. ${ }^{70}$ For example, Zhang et al. prepared a series of reactive PDMS-WPUs by using a novel PDMS bearing hydroxypropyl group at both chain ends and carbonyl groups at the side chain (Fig. 9). ${ }^{69}$ After post-added dihydrazide, self-crosslinking was achieved by the reaction between dihydrazide and carbonyl group during film formation, which resulted in higher mechanical properties.

Generally, PU materials exhibit relatively low thermal stability because of the presence of labile urethane groups (the scission of urethane groups is at $c a .230{ }^{\circ} \mathrm{C}$ ), and the degradation mechanism is very complex due to the variety of products formed in the process. ${ }^{70}$ TGA is a versatile method to evaluate their thermal stability. ${ }^{63}$ PDMS, with the thermal decomposition temperature around $500{ }^{\circ} \mathrm{C}$, has relatively higher thermal stability than WPU because the bond energy of Si-O $(451.4 \mathrm{~kJ}$ $\left.\mathrm{mol}^{-1}\right)$ is higher than that of $\mathrm{C}-\mathrm{C}\left(353.3 \mathrm{~kJ} \mathrm{~mol}^{-1}\right){ }^{17,57,67}$ Therefore, the incorporation of PDMS in WPU would result in the increase of thermal stability in the ultimate copolymer. ${ }^{47,66,75}$ For example, in the research of Wang et al., the initial degradation temperature of PDMS-WPU film with 15\% PDMS content rose by $20^{\circ} \mathrm{C}$ as compared to pure WPU, suggesting the role of PDMS is to prevent the degradation of PU backbone for the feasibility and facility of rotation along the $\mathrm{Si}-\mathrm{O}$ linkage can compel the PDMS molecule to absorb a part of heat energy..$^{53} \mathrm{In}$ another research, the char yield weight of PDMS-WPU at $700{ }^{\circ} \mathrm{C}$ was about $16 \%$ and that of WPU was about $3 \% .^{60}$

\section{Combined modification}

As mentioned in the Introduction section, a variety of technologies have been developed over the past decade in order to further improve the properties of WPU. Since the modification of WPU by PDMS has been demonstrated to be an effective method to enhance some key properties of WPU, combining this method with other technologies seems to be a feasible strategy to provide synergistic effect to obtain novel WPU with superior properties, which has drawn many researchers' attention. For instance, Zhang et al. prepared a UV-curable WPU nanocomposites based on PDMS and aqueous colloidal silica which resulted in simultaneously enhanced mechanical and surface properties of the nanocomposite films. ${ }^{66} \mathrm{Yu}$ et al. prepared a fluorinated PDMS-WPU which had lowest water absorption, higher water contact angles, and better mechanical/ thermal properties when the fluoride acrylic monomer content was $20 \mathrm{wt} \%$ and PDMS content was $3 \mathrm{wt} \%$, showing a promising application in waterproof coating. ${ }^{67}$ PDMS modified WPUacrylic hybrid emulsions and UV-curable PDMS modified WPUacrylate dispersions were also prepared and studied by different research groups. ${ }^{76-78}$ In view of the large number of existent modification technologies for WPU (which are still growing rapidly), effectively and creatively combining them with PDMSWPU to produce hybrid WPU that could meet specific requirements and find broader applications would become a research focus and need much efforts from many researchers.

\section{Conclusions and perspective}

PDMS-modified WPU could combine the advantages of PDMS with those of WPU, but the incompatibility problem should be overcome. Compared with physical modification, chemical modification receives much more research focus because it can increase the compatibility from the molecular level. Currently, four types of reactive PDMS are mainly used in the chemical modification of WPU. Different polymerization method leads to PDMS-WPU with different structure and different properties, and PDMS-grafted WPU may have a better surface property than that of PDMS-blocked WPU. This review classified PDMS-WPU as four types according to the type of hydrophilic segments present in the polymer chain, among which the anionic type is the most studied. For its preparation process, almost all published studies adopted the prepolymer mixing process. The effects of PDMS on colloidal, film-forming, surface, mechanical, and thermal properties as well as the effects of PDMS on microphase separation and hydrogen bonding behaviours of WPU were discussed in depth, with efforts to elucidate and understand these effects from the perspective of the "structuremorphology-property" relationships. Finally, combined modification of WPU via combining the PDMS modification with other effective modification technologies has become a new research trend, and several research examples were briefly reviewed.

As the chemistry and technology of WPU continue to develop and the appeal for a greener world becomes stronger as never before, the production of WPU has been steadily rising and its applications have significantly expanded. Considering the existence of several excellent reviews highlighting the recent progress in the applications of WPU, in which the current and potential application fields of PDMS-modified WPU are already contained, an Application section is therefore not included in this review and the reader should be referred to those reviews to obtain the in-depth coverage. ${ }^{\mathbf{1 7}, \mathbf{1 8 , 7 9 - 8 3}}$ Though the PDMSmodified WPU has made great progress in recent years and has already been commercialized in some fields like textile finishing, several aspects of challenges still remain. First, more types of reactive PDMS with different molecular weights, low cost, and high purity are needed for both industry and academia, which calls for more efforts from both researchers and engineers in silicone industry. Second, in order to satisfy 
the highly versatile and specific requirements for various end uses, the sole study of PDMS-modified WPU is not enough; rather, further study to compound with other appropriate additives should be conducted to obtain the final products. However, this kind of study is less seen in research papers. Third, as above discussed in the Combined Modification section, a challenge for many researchers is to creatively and effectively combine the PDMS modification technique with other modification technologies to produce novel WPU that have unique properties and can find broader applications. In the following decade, much more efforts is needed to realize the aim of building an PDMS-modified WPU industry which could provide better and greener products with lower cost and facile preparation. This review is anticipated to facilitate this process and encourage more future works to achieve this aim and to promote sustainability.

\section{Acknowledgements}

This work was supported by the Natural Science Foundation of Shanxi (No. 2015021081 and No. 201601D011019), the Project for Construction of Science and Technology Innovation Team of Shanxi (No. 2015013001-04), and the Chinese Innovation Fund for Technology Based Firms (No. 14C26211400582).

\section{References}

1 H. B. Chen, P. Shen, M. J. Chen, H. B. Zhao and D. A. Schiraldi, ACS Appl. Mater. Interfaces, 2016, 8, 3255732564.

2 H. C. Shi, D. Shi, L. G. Yin, Z. H. Yang, S. F. Luan, J. F. Gao, J. W. Zha, J. H. Yin and R. K. Y. Li, Nanoscale, 2014, 6, 1374813753.

3 X. L. Li, Z. Fang, X. Li, S. G. Tang, K. Zhang and K. Guo, New J. Chem., 2014, 38, 3874-3878.

4 Z. Fang, Z. Yang, D. Ji, N. Zhu, X. Li, L. Wan, K. Zhang and K. Guo, RSC Adv., 2016, 6, 90771-90776.

5 D. L. Tang, C. W. Macosko and M. A. Hillmyer, Polym. Chem., 2014, 5, 3231-3237.

6 P. K. Behera, K. M. Usha, P. K. Guchhait, D. Jehnichen, A. Das, B. Voit and N. K. Singha, RSC Adv., 2016, 6, 9940499413.

7 C. H. Su, Y. Q. Xu, F. Gong, F. S. Wang and C. F. Li, Soft Matter, 2010, 6, 6068-6071.

8 Q. W. Yong, F. W. Nian, B. Liao, L. P. Huang, L. Wang and H. Pang, RSC Adv., 2015, 5, 107413-107420.

9 H. Y. Mao, S. Y. Qiang, Y. Xu and C. X. Wang, New J. Chem., 2017, 41, 619-627.

10 K. Liu, Z. G. Su, S. D. Miao, G. H. Ma and S. P. Zhang, RSC Adv., 2016, 6, 31698-31704.

11 P. Alagi, R. Ghorpade, Y. J. Choi, U. Patil, I. Kim, J. H. Baik and S. C. Hong, ACS Sustainable Chem. Eng., 2017, 5, 38713881.

12 B. F. D'Arlas, L. Rueda, K. D. L. Caba, I. Mondragon and A. Eceiza, Polym. Eng. Sci., 2008, 48, 519-529.

13 M. M. Ding, J. H. Li, H. Tan and Q. Fu, Soft Matter, 2012, 8, 5414-5428.
14 S. H. Hsu, C. T. Hsieh and Y. M. Sun, J. Mater. Chem. B, 2015, 3, 9089-9097.

15 B. K. Kim, Colloid Polym. Sci., 1996, 274, 599-611.

16 D. K. Chattopadhyay and K. V. S. N. Raju, Prog. Polym. Sci., 2007, 32, 352-418.

17 O. Jaudouin, J. J. Robin, J. M. Lopez Cuesta, D. Perrin and C. Imbert, Polym. Int., 2012, 61, 495-510.

18 A. M. Nelson and T. E. Long, Macromol. Chem. Phys., 2014, 215, 2161-2174.

19 J. Zhao, T. Zhou, J. H. Zhang, H. M. Chen, C. Y. Yuan, W. D. Zhang and A. M. Zhang, Ind. Eng. Chem. Res., 2014, 53, 19257-19264.

20 N. G. Wang, L. N. Zhang and Y. S. Lu, Ind. Eng. Chem. Res., 2004, 43, 3336-3342.

21 Y. S. Lu and R. C. Larock, Biomacromolecules, 2007, 8, 31083114.

22 P. Florian, K. K. Jena, S. Allauddin, R. Narayan and K. V. S. N. Raju, Ind. Eng. Chem. Res., 2010, 49, 4517-4527.

23 Y. S. Lu and R. C. Larock, Biomacromolecules, 2008, 9, 33323340.

24 D. Y. Xie, F. Song, M. Zhang, X. L. Wang and Y. Z. Wang, Ind. Eng. Chem. Res., 2016, 55, 1229-1235.

25 S. Awad, H. Chen, G. Chen, X. Gu, J. L. Lee, E. E. Abdel-Hady and Y. C. Jean, Macromolecules, 2011, 44, 29-38.

26 L. H. Zhang, H. Zhang and J. S. Guo, Ind. Eng. Chem. Res., 2012, 51, 8434-8441.

27 X. D. Cao, H. Dong and C. M. Li, Biomacromolecules, 2007, 8, 899-904.

28 Y. Q. Li, W. B. Zhu, X. G. Yu, P. Huang, S. Y. Fu, N. Hu and K. Liao, ACS Appl. Mater. Interfaces, 2016, 8, 33189-33196.

29 M. Planes, J. Brand, S. Lewandowski, S. Remaury, S. Sole, C. Le Coz, S. Carlotti and G. Sebe, ACS Appl. Mater. Interfaces, 2016, 8, 28030-28039.

30 S. Seethapathy and T. Gorecki, Anal. Chim. Acta, 2012, 750, 48-62.

31 A. Dundua, S. Franzka and M. Ulbricht, Macromol. Rapid Commun., 2016, 37, 2030-2036.

32 J. K. Chen, H. W. Guo, P. Ding, R. Z. Pan, W. B. Wang, W. P. Xuan, X. Z. Wang, H. Jin, S. R. Dong and J. K. Luo, Nano Energy, 2016, 30, 235-241.

33 S. Vudayagiri and A. L. Skov, Polym. Adv. Technol., 2014, 25, 249-257.

34 J. R. Ebdon, D. J. Hourston and P. G. Klein, Polymer, 1984, 25, 1633-1639.

35 S. Vlad, A. Vlad and S. Oprea, Eur. Polym. J., 2002, 38, 829835.

36 R. Mieczkowski, Eur. Polym. J., 1992, 28, 53-55.

37 P. G. Klein, J. R. Ebdon and D. J. Hourston, Polymer, 1988, 29, 1079-1085.

38 J. R. Ebdon, D. J. Hourston and P. G. Klein, Polymer, 1986, 27, 1807-1814.

39 D. Klempner, H. L. Frisch and K. C. Frisch, J. Elastomers Plast., 1971, 3, 2-18.

40 M. S. Yen and P. Y. Tsai, J. Appl. Polym. Sci., 2010, 115, 35503558.

41 M. S. Yen and P. Y. Tsai, J. Appl. Polym. Sci., 2006, 102, 210221. 
42 M. S. Yen, P. Y. Tsai and P. D. Hong, Colloids Surf., A, 2006, 279, 1-9.

43 M. S. Yen and P. Y. Tsai, J. Appl. Polym. Sci., 2003, 90, 233243.

44 J. Kozakiewicz, Prog. Org. Coat., 1996, 27, 123-131.

45 Y. T. Yu, J. Wang and J. P. Zong, J. Adhes. Sci. Technol., 2015, 29, 861-874.

46 H. X. Song, Y. J. Luo, J. T. Luo, H. S. Lin and S. M. Qu, J. Shanghai Jiaotong Univ., 2006, 40, 1249-1252.

47 G. K. Zheng, M. Lu and X. P. Rui, Appl. Surf. Sci., 2017, 399, 272-281.

48 Y. T. Yu and J. Wang, Polym. Eng. Sci., 2014, 54, 805-811.

49 J. P. Zong, Q. S. Zhang, J. Y. Li, H. F. Sun, Y. T. Yu, S. J. Wang and Y. H. Liu, Polym. Mater. Sci. Eng., 2011, 27, 62-66.

50 Z. Wu, H. Wang, X. Tian, P. Cui, X. Ding and X. Ye, Phys. Chem. Chem. Phys., 2014, 16, 6787-6794.

51 M. M. Rahman, A. Hasneen, H.-D. Kim and W. K. Lee, J. Appl. Polym. Sci., 2012, 125, 88-96.

52 Y. H. Lee, E. J. Kim and H. D. Kim, J. Appl. Polym. Sci., 2011, 120, 212-219.

53 H. Wang, Y. Shen, G. Fei, X. Li and Y. Liang, J. Colloid Interface Sci., 2008, 324, 36-41.

54 X. Li, G. Fei and H. Wang, J. Appl. Polym. Sci., 2006, 100, 4046.

55 G. Fei, Y. Shen, H. Wang and Y. Shen, J. Appl. Polym. Sci., 2006, 102, 5538-5544.

56 C. H. Gao, J. N. Ni, Q. Zheng, S. L. Chen, W. W. Lin and J. G. Tu, Silicone Mater., 2009, 23, 357-362.

57 H. X. Song, Y. J. Luo, J. T. Luo, H. S. Lin and S. M. Qu, J. Chem. Eng., 2006, 57, 2486-2490.

58 G. Wang, C. Y. Hou, G. Z. Ma, T. T. Guan, L. X. Liang and X. Ji, Polyurethane Ind., 2015, 30, 5-9.

59 R. S. Chen, C. J. Chang and Y. H. Chang, J. Polym. Sci., Part A: Polym. Chem., 2005, 43, 3482-3490.

60 H. J. Naghash, I. Mohammadidehcheshmeh and M. Mehrnia, Polym. Adv. Technol., 2013, 24, 307-317. 61 S. C. Li and R. F. Yue, Paint Coat. Ind., 2010, 40, 1-3.

62 X. B. Wu, S. L. Wu, G. P. He and X. X. Li, Polyurethane Ind., 2009, 24, 21-24.
63 S. Ramesh, K. Tharanikkarasu, G. N. Mahesh and G. Radhakrishnan, J. Macromol. Sci., Part C: Polym. Rev., 1998, 38, 481-509.

64 Y. Xiao, X. Fu, Y. Zhang, Z. Liu, L. Jiang and J. Lei, Green Chem., 2016, 18, 412-416.

65 P. Krol, Prog. Mater. Sci., 2007, 52, 915-1015.

66 S. W. Zhang, Z. D. Chen, M. Guo, J. Zhao and X. Y. Liu, RSC Adv., 2014, 4, 30938.

67 F. F. Yu, X. Y. Xu, N. B. Lin and X. Y. Liu, $R S C A d v .$, 2015, 5, 72544-72552.

68 S. A. Madbouly, Y. Xia and M. R. Kessler, Macromolecules, 2013, 46, 4606-4616.

69 M. Zhang, Y. J. Wu, H. Y. Wu and Q. S. Zhang, J. Polym. Res., 2011, 19, 1-10.

70 X. Ji, Y. M. Zhou, B. L. Zhang, C. Y. Hou and G. Z. Ma, ISRN Polym. Sci., 2013, 2013, 1-10.

71 J. B. Dai, X. Y. Zhang, W. H. Li, Z. Ge, L. L. Feng and G. W. Xu, Polym. Mater. Sci. Eng., 2007, 23, 122-125.

72 Y. He, D. L. Xie and X. Y. Zhang, J. Mater. Sci., 2014, 49, 73397352.

73 W. T. Chen and S. C. Li, Paint Coat. Ind., 2005, 35, 13-15.

74 J. Yang, Y. L. Gao, J. H. Li, M. M. Ding, F. Chen, H. Tan and Q. Fu, RSC Adv., 2013, 3, 8291.

75 Y. J. Li, S. Chen and F. Wu, Asian J. Chem., 2014, 26, 57035708.

76 H. D. Hwang and H. J. Kim, React. Funct. Polym., 2011, 71, 655-665.

77 C. Y. Zhang, X. Y. Zhang, J. B. Dai and C. Y. Bai, Prog. Org. Coat., 2008, 63, 238-244.

78 C. Y. Bai, X. Y. Zhang, J. B. Dai and J. H. Wang, J. Coat. Technol. Res., 2008, 5, 251-257.

79 A. Shirke, B. Dholakiya and K. Kuperkar, Polym. Sci., Ser. B, 2015, 57, 292-297.

80 J. O. Akindoyo, M. D. H. Beg, S. Ghazali, M. R. Islam, N. Jeyaratnam and A. R. Yuvaraj, RSC Adv., 2016, 6, 114453-114482.

81 A. Noreen, K. M. Zia, M. Zuber, S. Tabasum and M. J. Saif, Korean J. Chem. Eng., 2015, 33, 388-400.

82 V. D. Athawale and R. V. Nimbalkar, J. Am. Oil Chem. Soc., 2010, 88, 159-185.

83 G. A. Howarth, Surf. Coat. Int., Part B, 2003, 86, 111-118. 